\title{
Nonuniversal Critical Spreading in Two Dimensions
}

\author{
Ronald Dickman ${ }^{\dagger}$ \\ Department of Physics and Astronomy, Lehman College, CUNY, Bronx, NY 10468-1589
}

(December 19, 2018)

\begin{abstract}
Continuous phase transitions are studied in a two dimensional nonequilibrium model with an infinite number of absorbing configurations. Spreading from a localized source is characterized by nonuniversal critical exponents, which vary continuously with the density $\phi$ in the surrounding region. The exponent $\delta$ changes by more than an order of magnitude, and $\eta$ changes sign. The location of the critical point also depends on $\phi$, which has important implications for scaling. As expected on the basis of universality, the static critical behavior belongs to the directed percolation class.
\end{abstract}

PACS numbers: 05.50.+q, 02.50.-r, 05.70.Ln

${ }^{\dagger}$ e-mail address: dickman@lcvax.lehman.cuny.edu 


\section{INTRODUCTION}

Despite recent progress, understanding the phase diagrams and critical points of nonequilibrium models remains a challenging problem. In the relatively wellunderstood case of continuous transitions into an absorbing state (i.e., one that traps the dynamics), a high degree of universality has been found, supporting the prediction that such transitions belong generically to the class of directed percolation (DP) [1 [3]. Examples include the basic contact process and its variants [甘] 9], surface reaction models [3, 10,11], branching annihilating random walks with odd parity [12 14], and assorted multiparticle processes [15] 19]. The static critical behavior of models with multiple absorbing configurations 20 27 also falls in the DP class. On the other hand, some aspects of time-dependent critical behavior in the latter class of models are surprising: the critical exponents which describe spreading from a seed vary continuously with the density in the environment, and the usual hyperscaling relation must be modified. Until now this phenomenon has been investigated in one dimension only; here I present the first study of nonuniversal critical spreading in a two dimensional model.

Models with an absorbing state are characterized by an order parameter $\rho$ - the density of self-reproducing entities or active sites. The stationary value $\bar{\rho}$ vanishes as the reproduction rate $\lambda$ approaches a critical value $\lambda_{c}: \bar{\rho} \propto \Delta^{\beta}$ for small $\Delta=\lambda-\lambda_{c}$. For $\lambda<\lambda_{c}$ the only steady state is the absorbing state, in which all change ceases. Much insight is gained from studies of spreading from a single active site [28]. The chief quantities of interest are the survival probability, $P(t)$, (the process is said to survive so long as it has not become trapped in the absorbing state), the mean 
number of active sites $n(t)$, and the mean-square distance, $R^{2}(t)$, of active sites from the original seed. At the critical point they follow asymptotic power laws,

$$
\begin{aligned}
& P(t) \propto t^{-\delta}, \\
& n(t) \propto t^{\eta},
\end{aligned}
$$

and

$$
R^{2}(t) \propto t^{z}
$$

These power laws, which form the most salient feature of the critical spreading process, have been confirmed for a broad class of models. The exponents $\delta, \eta$, and $z$, estimated from simulations and series expansions [29] are in good agreement with DP values.

Several models have been devised with absorbing states that embrace a large number of configurations: two-dimensional catalysis models 22 25], and one-dimensional particle systems such as the pair contact process (PCP) 20, 21,27. In the PCP each site of a lattice $(\mathbf{Z})$ is either vacant or occupied by a particle; the active "sites" are nearest-neighbor particle pairs. They are annihilated with probability $p$, and give birth, with probability $1-p$, to a new particle at a vacant neighboring site. Since particles may only be created or destroyed if there are pairs, and since particles cannot move to other sites, any configuration of isolated particles is absorbing; the system always becomes trapped in such a configuration if $p>p_{c}$. While static critical behavior in models with multiple absorbing configurations still conforms to DP [30], spreading presents a new feature: one can choose the absorbing configuration into which the seed is placed. Critical spreading has been studied in three rather different one-dimensional models with multiple absorbing configurations: the PCP, the 
threshold transfer process, and the dimer reaction [21,27]. For "natural" absorbing configurations (those generated by the critical process itself), the spreading exponents assume DP values. If, however, the initial density $\phi_{\mathrm{i}}$ differs from the natural value, the exponents $\delta$ and $\eta$ change; they vary linearly with $\phi_{\mathrm{i}}$. ( $\phi$ represents the density of isolated particles in the PCP, not the order parameter.) A new exponent, $\beta^{\prime}$ must be introduced to describe the ultimate survival probability:

$$
P_{\infty} \equiv \lim _{t \rightarrow \infty} P(t) \propto\left(p_{c}-p\right)^{\beta^{\prime}}
$$

in the active regime. This exponent also depends on $\phi_{\mathrm{i}} \cdot\left(\beta^{\prime}=\beta\right.$ for natural initial configurations, just as for models with a unique absorbing configuration.) It appears in the generalized hyperscaling relation [27,

$$
\left(1+\frac{\beta}{\beta^{\prime}}\right) \delta+\eta=\frac{d z}{2},
$$

which reduces to the relation originally derived by Grassberger and de la Torre [28] for $\beta^{\prime}=\beta$. Certain properties are independent of $\phi_{\mathrm{i}}$ : the location of the critical point, the exponent $z$, and $\delta+\eta$, which governs population growth in surviving trials. This suggests that the initial density influences the survival probability, reflected in the exponents $\delta$ and $\beta^{\prime}$, but does not alter the asymptotic properties of surviving trials.

The background sketched above motivates the investigation of critical spreading in dimensions $\geq 2$, which offers a greater range of possible growth patterns. In the following section I define a two-dimensional model with multiple absorbing configurations, and describe the simulation procedure used to study it. Results are presented in Sec. III, and a scaling analysis in Sec. IV, followed by a brief summary in Sec. V. 


\section{THE MODEL}

The second neighbor reaction (SNR) is a Markov process or interacting particle system in which each site of the square lattice $\mathbf{Z}^{2}$ is either vacant or occupied by a particle. Particles may not occupy adjacent sites. If all four nearest neighbors of a vacant site $(\mathrm{i}, \mathrm{j})$ are also vacant $(\mathrm{i}, \mathrm{j})$ is said to be open. Open sites are the only active sites in the model. In each step of the process, a site is chosen at random; if it is open, a particle is placed there provisionally, otherwise nothing happens. Suppose a particle has just arrived at $(\mathrm{i}, \mathrm{j})$. If all four of the second neighbors ( $\mathrm{i} \pm 1, \mathrm{j} \pm 1)$ are vacant the new particle is adsorbed, and remains at $(\mathrm{i}, \mathrm{j})$. But if any of the second neighbors are occupied, the new particle is only adsorbed with probability $p$; with probability $1-p$ it reacts with one of its neighbors (selected at random, if necessary), and both particles leave the lattice. Each such reaction creates one or more new open sites. Adsorbed particles are immobile, and can only leave the surface in a reaction with a newly-arrived particle. Each adsorption attempt marks a fixed time interval; we take the time unit as $N=L^{2}$ such events.

Since the fraction of open sites is quite small in the vicinity of the critical point, efficiency is greatly improved by choosing trial adsorption sites from a list of open sites. This entails a variable time increment, $\Delta t=1 / N_{o}$, for each event, where $N_{o}$ is the number of open sites. Taking statistics at fixed time intervals (rather than after a fixed number of events), ensures proper stationary averages.

For $p=1$ the model corresponds to random sequential adsorption (RSA) with nearest-neighbor exclusion; starting from an empty lattice, particles adsorb until no open sites remain. In this case there is an exponential approach to the jamming 
density $\phi_{\infty}=0.36413$ [31, 32]. The order parameter $\rho$ for the SNR is the fraction of open sites; configurations devoid of open sites are absorbing. Of course, many absorbing configurations are possible, with particle densities $\phi$ ranging from $1 / 5$ to 1/2 (see Fig. 5). I shall refer to the set of absorbing configurations characterized by common statistical properties (particle density and correlations) as an absorbing state. It is also useful to define the ensemble $\mathcal{A}_{p, L}$ of absorbing configurations generated by the SNR with adsorption probability p, starting from an empty lattice of size $L$. For any absorbing configuration $\zeta$ on $L^{2}$ sites, $\mathcal{A}_{p, L}(\zeta)$ is the probability of realizing $\zeta$ as the final configuration.

Based upon experience with models of this kind - in particular, with the dimer reaction, the one-dimensional cousin of the SNR [21,27] — one expects that there is a critical value $p_{c}$ above which the system always gets trapped, whilst for $p<p_{c}$ there is an active state in which the reaction may proceed indefinitely. Equivalently, one expects that for $p>p_{c}$ the absorbing state is the only stationary state, and that for $p<p_{c}$ there is in addition a nontrivial invariant measure on configuration space. (While the only latter emerges in the infinite-size limit, for $p<p_{c}$ the quasi-stationary state is sufficiently long-lived to allow precise characterization in simulations.)

\section{SIMULATION RESULTS}

An accurate estimate of $p_{c}$ is crucial for reliable determination of critical behavior. Several simulation methods are available for locating the critical point: half-life studies, time-dependent simulations, and analysis of stationary properties. Use of any one method exclusively can be misleading. Here I begin with the half-life method, which 
is based on finite-size scaling analysis of the mean survival time. One generates a large number of independent realizations, all starting from an empty lattice (all sites open), with periodic boundaries, and determines the time $\tau$ required for half the sample to reach the absorbing state. $\tau$ is a decreasing function of $p$ and an increasing function of lattice size $L$. One expects that in the vicinity of the critical point 15

$$
\tau(p, L) \simeq L^{\nu_{\|} / \nu_{\perp}} h\left(\Delta L^{1 / \nu_{\perp}}\right)
$$

where $\Delta=p_{c}-p$ and $h$ is a scaling function. The exponents $\nu_{\|}$and $\nu_{\perp}$ govern the divergence of the relaxation time $t_{r}$ and the correlation length $\xi$ as $\Delta \rightarrow 0$ : $t_{r} \propto \Delta^{-\nu_{\|}}$and $\xi \propto \Delta^{-\nu_{\perp}}$. The critical point is distinguished by the simple powerlaw $\tau \propto L^{\nu_{\|} / \nu_{\perp}}$ as $L \rightarrow \infty$. Following a preliminary survey which indicated that $p_{c} \simeq 0.3935$, I estimated $\tau$ for $L=16,32,4864,96,128$, and 176 , for $p=0.3930$ - 0.3940. It is convenient to plot $\ln \tau-\left(\nu_{\|} / \nu_{\perp}\right) \ln L$ versus $\ln L$, using the expected value of the exponent ratio for DP in $2+1$ dimensions (see Fig. 1). Only for $p$ in the range $0.3926-0.3927$ are the data consistent with a power law. The corresponding exponent is $\nu_{\|} / \nu_{\perp}=1.76(2)$, in good agreement with the DP value, 1.764(26).

More precise estimates of $p_{c}$ may be obtained from simulations of spreading [28]. As noted above, the critical point is marked by asymptotic power laws, Eqs. (11) - (3). This part of the study focuses on the natural absorbing state - configurations drawn from $\mathcal{A}_{p_{c}, L}$, defined in Sec. II. Initial configurations are generated by running the simulation (starting with all sites vacant), at a given $p$, on a lattice of size $L^{\prime}=128$, until it reaches an absorbing configuration. Following the procedure of Ref. [21, copies of this configuration are assembled into an absorbing configuration of size $L=1280$. Then a single (randomly chosen) particle is removed, creating an open site 
(or possibly a small cluster of open sites), which serves as the seed for the spreading process.

In spreading simulations the process halts before reaching the boundary; there are no finite-size effects as such, though one does of course expect finite-time corrections to scaling. Trials ran to a maximum time $t_{\max }=10^{4} ; 5 \times 10^{4}$ independent trials were generated for each $p$ value studied. The survival probability, mean number of open sites, and mean-square spread for $p=0.392615$ are plotted in Fig. 2, showing clear evidence for power laws. In order to fix $p_{c}$ and the exponents with precision, I plot, in Fig. 3, the local slopes of the $\log$ - log graphs versus $1 / t$, for several values of $p$. (The local slope $\delta_{t}$ is found from a linear least-squares fit to the data for $\ln P(t)$ over the range $[\ln t-1.5, \ln t+1.5]$, and similarly for the other exponents.) I conclude that $0.39261<p_{c}<0.39263$, given the marked curvatures of the $\delta_{t}$ and $\eta_{t}$ graphs for $p$-values outside this range. The exponents obtained by extrapolating the local slopes to infinite- $t$ are in good agreement with accepted values for DP in $2+1$ dimensions (see Table I).

I turn to the exponent $\beta^{\prime}$ governing the ultimate survival probability starting from a localized active region. Natural absorbing configurations, constructed as above (with $p=p_{c}=0.39262$ ), were used in determinations of $P_{\infty}$ for $p<p_{c}$. These studies yield $\beta^{\prime}=0.58(2)$, whilst in DP $\beta=\beta^{\prime}=0.586(14)$ 33. The stationary open site density $\bar{\rho}$ was determined on lattices of size $L=16,32,64$, and 128 . To minimize finite size effects, $\bar{\rho}(p)$ was obtained for two or more system sizes, until doubling $L$ produced no significant change. A linear least-squares fit to a plot of $\ln \rho$ versus $\ln \left(p_{c}-p\right)$, (with $\left.p_{c}=0.39262\right)$, yields $\beta=0.56(2)$. In summary, the static critical behavior and critical spreading from "natural" absorbing configurations are both fully 
consistent with DP scaling in $2+1$ dimensions.

It is of interest to characterize the natural absorbing configurations. Large samples of the latter were generated by running the simulation at $p_{c}$, starting from an open lattice $(L \leq 176)$, until it reached an absorbing configuration. The results for the particle density $\phi_{\text {nat }}$ show only a weak dependence on $L$, permitting reliable extrapolation to infinite- $L$, yielding $\phi_{\text {nat }}=0.33429(3)$. Further insight into absorbing configurations is provided by the two-point total correlation function,

$$
h(\mathbf{r}) \equiv \frac{\langle\sigma(\mathbf{x}) \sigma(\mathbf{x}+\mathbf{r})\rangle}{\phi^{2}}-1,
$$

where $\sigma(\mathbf{x})$ is an indicator variable which is 1 iff site $\mathbf{x}$ is occupied. Fig. 4 shows that $h(r)$ is negligible for $r>6$. Thus correlations in natural absorbing configurations in the SNR are of only slightly greater range than in the one-dimensional dimer reaction 21].

Having established the properties of the active stationary state, the natural absorbing state, and of critical spreading from the latter, I turn to spreading in "nonnatural" absorbing configurations. Detailed studies were performed for the maximal density $(\phi=0.5)$, the minimal one $(\phi=0.2)$, and for two kinds of configurations with $\phi=0.25$, one with square-lattice symmetry, the other having a preferred axis. In addition to these periodic states, absorbing configurations were generated via RSA. (Examples are shown in Fig 5.) In each case, the critical point, $p_{c}(\phi)$, was identified by searching for asymptotic power-laws as in Eqs (11) - (3).

Consider first the lowest density absorbing configuration $(\phi=0.2)$, in which particles are arrayed in a $\sqrt{5} \times \sqrt{5}$ pattern. Simulations indicate that the spreading process is supercritical for $p=p_{c, b u l k}=0.39262$. The survival probability $P(t)$, for 
example, tends to a nonzero value as $t \rightarrow \infty$, rather than exhibiting power-law decay. Power-law spreading occurs for $p=0.4102(2)$, about $4 \%$ greater than $p_{c, b u l k}$ (see Fig 6). As indicated in Table I, the spreading exponents $\eta$ and $z$ are much larger than the corresponding DP values, and $\delta$ and $\beta^{\prime}$ are much smaller. The changes in $p_{c}$ and in the exponents accord with the notion that open sites spread more readily in a region of low particle density. 


\section{TABLES}

TABLE I. Critical point and exponents in the second neighbor reaction. Figures in parentheses denote uncertainties.

\begin{tabular}{|l|l|l|l|l|l|}
\hline \hline$\phi$ & $p_{c}$ & $\delta$ & $\eta$ & $z$ & $\beta^{\prime}$ \\
\hline 0.2 & $0.4102(2)$ & $0.078(8)$ & $0.64(4)$ & $1.84(2)$ & $0.125(5)$ \\
0.25 (isotropic) & $0.3965(1)$ & $0.054(2)$ & $0.90(1)$ & $1.76(1)$ & $0.18(5)$ \\
0.25 (aniso.) & $0.39238(2)$ & $0.677(5)$ & $-0.035(5)$ & $0.97(1)$ & \\
0.33443 (natural) & $0.39262(1)$ & $0.468(6)$ & $0.216(8)$ & $1.117(13)$ & $0.58(1)$ \\
0.36413 (RSA) & $0.39240(5)$ & $0.64(1)$ & $0.035(20)$ & $1.038(3)$ & $0.70(2)$ \\
0.5 & $0.39220(5)$ & $1.30(5)$ & $-0.12(6)$ & $0.91(2)$ & $2.0(2)$ \\
\hline $\mathrm{DP}$ & - & $0.460(6)$ & $0.214(8)$ & $1.134(4)$ & $0.586(14)$ \\
\hline \hline
\end{tabular}


The absorbing configuration with maximal particle density $(\phi=1 / 2)$, by contrast, presents hostile terrain for spreading. Running at the bulk critical point, the process appears to be subcritical: $P(t)$ and $n(t)$ decay exponentially. To observe power-law spreading one must reduce $p$ to 0.3922 , about $0.14 \%$ below the bulk critical value. The shift in $p_{c}$ is small, but the effect on the exponents is dramatic: spreading is sub-diffusive $(z<1)$, and $\eta$ is negative! (The latter is consistent with known constraints: $\eta+\delta \geq 0$, so the population in surviving trials increases with time.) Studies with intermediate initial densities, summarized in Table I and Fig. 7, confirm the tendencies noted for the extreme cases: higher densities correspond to smaller values of $\eta$ and $z$, larger $\delta$ and $\beta^{\prime}$, and a depressed $p_{c}$, and conversely, consistent with the expectation that the larger $\phi$, the more spreading is impeded. The only exception is the anisotropic system, which despite having $\phi<\phi_{\text {nat }}$, resembles isotropic systems with $\phi>\phi_{\text {nat }}$. Why anisotropy renders spreading more difficult is unclear. The contrast between the two studies at $\phi=0.25$ is nonetheless striking, and demonstrates that factors other than the particle density can influence critical spreading.

\section{SCALING THEORY}

We have seen that as $\phi$, the density of particles in the environment, is varied, the location of the critical point changes as well. The shift in $p_{c}$ implies that critical processes starting from non-natural absorbing configurations do not evolve to the bulk critical state, which exists uniquely at $p_{c, b u l k}$. As a result, the usual scaling theory for absorbing states must be modified. In (isotropic) absorbing configurations with $\phi<\phi_{n a t}$, critical spreading occurs at $p=p_{c}>p_{c, \text { bulk }}$, where an active steady 
state is not possible. As the population of open sites spreads, it leaves in its wake a region devoid of open sites, with particle density $\phi_{f}$, slightly greater than $\phi_{\text {nat }}$. After some time, this region cannot be reactivated. (For $p>p_{c, n a t}$, the survival probability decays exponentially even when $\phi=\phi_{\text {nat }}$.) Thus for $p_{c, n a t}<p<p_{c}(\phi)$, the active region is confined to an expanding ring; it is a kind of "chemical wave" which converts one type of absorbing configuration to another as it passes. An example of this kind of spreading is shown in Fig 8. The mean density of open sites (averaged over 500 trials), is plotted in Fig. 9 as a function of distance from the origin, confirming that the active region forms an expanding ring. Critical spreading with $\phi>\phi_{\text {nat }}$ presents a rather different picture. Here the critical point lies below $p_{c, b u l k}$, which means that surviving trials consist of an expanding region with a small but finite density of open sites.

For non-natural initial conditions, the exponents violate the hyperscaling relation, Eq (5). This is not surprising, since the scaling argument assumes that surviving processes evolve, for large $t$, into a bulk critical system. [27,28] This assumption, as we have seen, is not valid when $p_{c} \neq p_{c, b u l k}$. To describe this novel situation, the scaling analysis can be modified as follows. We assume, as usual, that spreading may be described in terms of a pair of scaling functions, defined via 28]

$$
\rho(x, t) \sim t^{\eta-d z / 2} G\left(x^{2} / t^{z}, \Delta t^{1 / \nu_{\|}}\right)
$$

and

$$
P(t) \sim t^{-\delta} \Phi\left(\Delta t^{1 / \nu_{\|}}\right)
$$

where $\Delta=p_{c}(\phi)-p$ is the distance from the critical point. (For $\Delta>0$ spreading may continue indefinitely.) In Eq. (8) $\rho(x, t)$ is the local order-parameter density, 
averaged over all realizations; it is concentrated near the origin at time zero. The prefactors are constructed to yield power laws - Eqs. (1) - (3) - when $\Delta=0$. Existence of the limit $P_{\infty}$ implies that $\Phi(x) \sim x^{\beta^{\prime}}$ as $x \rightarrow \infty$, with $\beta^{\prime}=\delta \nu_{\|}$.

Consider spreading with $\phi<\phi_{\text {nat }}$ and $p_{c}(\phi)>p_{c, b u l k}$, in which the active region is an expanding ring. Simulations indicate that for $\Delta>0$, the active region expands at a constant speed $v(\Delta)$, and that the maximum open-site density in surviving trials attains a steady value $\rho_{o}(\Delta)$ for large $t$. In the contact process and similar models, $\rho(\mathbf{x}, t) \rightarrow P_{\infty} \bar{\rho}$ as $t \rightarrow \infty$, for any fixed $\mathbf{x}$. But here we expect instead that $\rho(\mathbf{x}, t) \rightarrow P_{\infty} \rho_{o}(\Delta) f(|\mathbf{x}| / v t)$, where $f(u)$ attains its maximum value (unity) at $u_{0} \approx 1$, and $f \rightarrow 0$ as $u \rightarrow 0$ or $u \rightarrow \infty$. We expect $\rho_{0}$ to have a power-law dependence on $\Delta$ :

$$
\rho_{0} \sim \Delta^{\beta^{\prime \prime}}
$$

Consider the limit $t \rightarrow \infty,|\mathbf{x}| \rightarrow \infty$, with $|\mathbf{x}| / v t \approx u_{0}$. For $\Delta$ small but positive, $\rho(\mathbf{x}, t) \sim \Delta^{\beta^{\prime}} \rho_{0}$, so that $G(\infty, y) \sim y^{\beta^{\prime}+\beta^{\prime \prime}}$ for large $y$. On the other hand, we must have $G(\infty, y) \sim y^{-\nu_{||}(\eta-d z / 2)}$ for $\lim _{|\mathbf{x}|, t \rightarrow \infty} \rho(\mathbf{x}, t)$ to exist. Comparing these asymptotic behaviors, we find a hyperscaling relation for annular spreading

$$
\left(1+\frac{\beta^{\prime \prime}}{\beta^{\prime}}\right) \delta+\eta=\frac{d z}{2}
$$

Inserting the exponent values shown in Table 1 yields $\beta^{\prime \prime}=1.80(35)$, for $\phi=0.2$. This is consistent with $\beta^{\prime \prime}=1.68(6)$, obtained directly from simulations at $p$-values between $p_{c, b u l k}$ and $p_{c}(0.2)$.

As noted above, surviving trials at $p_{c}(\phi)$ for $\phi>\phi_{\text {nat }}$ develop a finite open-site density and so are compact objects. The critical exponents for spreading of compact colonies are expected to satisfy the relation [34: 


$$
\delta+\eta=\frac{d z}{2}
$$

which simply expresses the growth of population in a region of positive density, given that the radius grows $\sim t^{z / 2}$. The exponents for density $\phi=0.5$ are, however, inconsistent with Eq. (12): $\delta+\eta-z=0.27(13)$ (The deviation from the generalized DP hyperscaling relation, Eq. (5), is considerably larger: $\left(1+\beta / \beta^{\prime}\right) \delta+\eta-z=0.6(2)$.) For RSA initial configurations $(\phi=0.36413)$ the corresponding deviations are $-0.3(1)$ and $0.2(1)$. We can understand this discrepancy by noting that the onset of compact growth, which requires that the colony diameter $D>>\xi$, the bulk correlation length, should only occur at very long times, since $\left|p-p_{c, b u l k}\right| / p_{c, b u l k}$ is very small (less than $\left.10^{-3}\right)$. This is supported by the observation that even at fairly late times $\left(t=10^{4}\right)$, the distribution of open sites does not appear compact, but rather consists of several disconnected regions (see Fig. 10). Presumably the spreading exponents found for $\phi=0.5$ and 0.364 are not the asymptotic ones; their determination will require studies of considerably longer duration.

\section{SUMMARY}

The first study of nonuniversal spreading in two dimensions reveals a large variation of critical exponents with the density $\phi$ of the environment into which the process grows. The variation of the spreading exponents is much more pronounced than in one-dimensional models with multiple absorbing configurations [21,27]. In the SNR $\delta$ and $\beta^{\prime}$ vary by more than an order of magnitude, $\eta$ changes sign, and $z$, which is essentially constant in the one-dimensional models, varies by more than a factor of two. (Similarly, $\delta+\eta$ appears constant in one dimension, but varies substantially here.) 
Moreover, the critical point, $p_{c}$, depends on $\phi$, whereas in one dimension it remains constant. The shift in $p_{c}$ has important consequences for scaling since the critical spreading process does not evolve into a bulk critical state for $\phi \neq \phi_{\text {nat }}$. Comparison of spreading in two environments with the same density but different symmetries reveals that the process is strongly influenced by factors other than the density.

\section{ACKNOWLEDGMENTS}

I thank Geoff Grinstein and Miguel Angel Muñoz for helpful discussions. Simulations employed the facilities of the University Computing Center, City University of New York. 


\section{REFERENCES}

[1] H. K. Janssen, Z. Phys. B 42, 151 (1981).

[2] P. Grassberger, Z. Phys. B 47, 365 (1982).

[3] G. Grinstein, Z.-W. Lai, and D. A. Browne, Phys. Rev. A 40, 4820 (1989).

[4] T. E. Harris, Ann. Prob. 2, 969 (1974).

[5] T. M. Liggett, Interacting Particle Systems (Springer-Verlag, New York, 1985).

[6] R. Durrett, Lecture Notes on Particle Systems and Percolation (Wadsworth Pub. Co., Pacific Grove, CA, 1988).

[7] N. Konno, Phase Transitions of Interacting Particle Systems (World Scientific, Singapore, 1994).

[8] I. Jensen and R. Dickman, J. Phys. A. 26, L151 (1993).

[9] I. Jensen and R. Dickman, Physica A 203, 175 (1994).

[10] R.M. Ziff, E. Gulari, and Y. Barshad, Phys. Rev. Lett. 56, 2553 (1986).

[11] I. Jensen, H. C. Fogedby, and R. Dickman, Phys. Rev. A 41, 3411 (1990).

[12] H. Takayasu and A. Yu. Tretyakov, Phys. Rev Lett. 68, 3060 (1992).

[13] I. Jensen, Phys. Rev. E 47, 1 (1993).

[14] I. Jensen, J. Phys. A 26, 3921 (1993).

[15] T. Aukrust, D. A. Browne, and I. Webman, Europhys. Lett. 10, 249 (1989); Phys. Rev. A 41, 5294 (1990). 
[16] R. Bidaux, N. Boccara, and H. Chaté, Phys. Rev. A39, 3094 (1989).

[17] R. Dickman, Phys. Rev. A 42, 6985 (1990).

[18] R. Dickman and T. Tomé, Phys. Rev. A 44, 4833 (1991).

[19] H. Park, J. Köhler, I.-M. Kim, D. ben-Avraham, and S. Redner, J. Phys. A26, 2071 (1993).

[20] I. Jensen, Phys. Rev. Lett. 70, 1465 (1993).

[21] I. Jensen and R. Dickman, Phys. Rev. E 48, 1710 (1993).

[22] E. V. Albano, J. Phys. A: Math. Gen. 25, 2557 (1992).

[23] D. ben-Avraham and J. Köhler, J. Stat. Phys. 65, 839 (1992).

[24] K. Yaldram, K.M. Khan, N. Ahmed, N. and M.A. Khan, J. Phys. A: Math. Gen. 26, L801 (1993).

[25] I. Jensen, J. Phys. A: Math. Gen. 27, L61 (1994).

[26] I. Jensen, Int. J. Mod. Phys. B 23, 3299 (1994).

[27] J. F. F. Mendes, R. Dickman, M. Henkel and M. C. Marques, J. Phys. A: Math. Gen. 27, 3019 (1994).

[28] P. Grassberger and A. de la Torre, Ann. Phys. (NY) 122, 373 (1979).

[29] I. Jensen and R. Dickman, J. Stat. Phys. 71, 89 (1993).

[30] M.A. Muñoz, R. Dickman, G. Grinstein, and R. Livi, Phys. Rev. Lett., in press.

[31] P. Meakin, J.L.Cardy, E. Loh, and D.J. Scalapino, J. Chem. Phys. 86, 2380 
(1987).

[32] R. Dickman, J.-S. Wang, and I. Jensen, J. Chem. Phys. 94, 8252 (1991).

[33] R.C. Brower, M.A. Furman, and M. Moshe, Phys. Lett. B76, 213 (1978).

[34] R. Dickman and A. Yu. Tretyakov, Phys. Rev. E52, 3218 (1995). 


\section{Figure Captions}

Fig. 1. Dependence of the half-life, $\tau$, on system size. $\nu_{\|} / \nu_{\perp}=1.764$, as expected for DP in $2+1$ dimensions. Filled squares: $p=0.3928 ; \square: p=0.3927 ; \times: p=0.3926 ; \diamond$ : $p=0.3925$.

Fig. 2. Survival probability, mean number of open sites, and mean-square spread for natural initial conditions, $p=0.392615$.

Fig. 3. Local slopes, $\delta_{t}(\mathrm{a}), \eta_{t}(\mathrm{~b})$, and $z_{t}(\mathrm{c})$, for natural initial conditions. $\diamond$ : $p=0.39261 ; \square: 0.392615 ; \times: 0.39262 ;+: 0.39263$.

Fig. 4. Two-point correlation function $h(r)$ in natural absorbing configurations. • $(1,0)$ direction; $\times:(1,1)$ direction; $\circ:(2,1)$ direction.

Fig. 5. Examples of initial absorbing configurations investigated in this work. Left: upper, density $\phi=1 / 5$; mid, $\phi=1 / 4$, anisotropic; lower, $\phi=0.363$, (RSA); right: upper, $\phi=0.328$, (natural); mid, $\phi=0.25$, isotropic; lower, $\phi=0.5$.

Fig. 6. Local slopes, $-\delta_{t}(\mathrm{a}), \eta_{t}(\mathrm{~b})$, and $z_{t}(\mathrm{c})$, for $\phi=0.2 . \quad+: p=0.4106$; $\square$ : $p=0.4104 ; \diamond: p=0.4102 ; \times: p=0.4100 ; \circ: p=0.4096$.

Fig. 7. Spreading exponents $\delta, \eta, z$, and $\beta^{\prime}$ vs initial density $\phi$.

Fig. 8. Spread of open sites, $\phi=0.2, p=p_{c}$. Light gray: $t=200$; dark gray: $t=500$; black: $t=1000$. 
Fig. 9. Mean density of open sites vs distance from seed, for $\phi=0.2, p=p_{c}$. From left to right, $t=200,500$, and 1000 .

Fig. 10. A typical colony for $\phi=0.5, p=p_{c}, t=10^{4}$. The line represents 100 lattice spacings. 